\title{
Ro 20-1724 Ameliorates Learning Deficit and Long-Term Memory Impairment Secondary to Repeated Ketamine Anesthesia in Young Rats
}

\author{
Sheng Peng ${ }^{1}$, Hai Yan Sun ${ }^{2}$, Gong-Jian Liu ${ }^{3}$, Xia Yang ${ }^{4}$, George Mychaskiw II $^{5^{*}}$ \\ ${ }^{1}$ Department of Anesthesiology, Soochow University, Wuxi, China; ${ }^{2}$ Department of Anesthesiology, Traditional Chinese Medicine \\ Hospital of Zhang Jiang, China; ${ }^{3}$ Department of Anesthesiology, Xuzhou Medical College, Xuzhou, China; ${ }^{4}$ Department of Anesthe- \\ siology, Tengzhou Central People's Hospital, Tengzhou, China; ${ }^{5}$ Department of Anesthesiology, Nemours Children's Hospital, Or- \\ lando, USA. \\ Email: "gmychask@nemours.org
}

Received May 20 ${ }^{\text {th }}, 2013$; revised June $25^{\text {th }}, 2013$; accepted July $4^{\text {th }}, 2013$

Copyright (C) 2013 Sheng Peng et al. This is an open access article distributed under the Creative Commons Attribution License, which permits unrestricted use, distribution, and reproduction in any medium, provided the original work is properly cited.

\begin{abstract}
To investigate effects and possible mechanism of Ro 20-1724, a PDE4 inhibitor, on long-time learning and memory ability following repeated ketamine exposure in immature rats. Methods: Sixty 21-day-old SD rats were randomly divided into five groups $(\mathrm{n}=12)$ : C: Normal control group, S: Saline control group, K: Ketamine, $\mathrm{K}+\mathrm{Ro}$ : Ketamine + Ro 20-1724, K + E: Ketamine + ethanol vehicle. Ro 20-1724 $\left(0.5 \mathrm{mg} \cdot \mathrm{kg}^{-1}\right)$ or its vehicle (ethanol) was administered intraperitoneally 30 minutes after ketamine anesthesia $\left(70 \mathrm{mg} \cdot \mathrm{kg}^{-1}\right)$, daily for seven days. Nine weeks after birth, the Morris water maze was used to test the ability of learning and spatial localization memory on the rats. Following behavioral testing, animals' hippocampi were removed for Western blot and electron microscopic examination. Results: In the Morris water maze test, compared with controls, the escape latency in groups exposed to ketamine or ketamine plus the ethanol vehicle were significantly prolonged $(P<0.05)$, the ability of spatial localization was lower $(P<0.05)$, and the expression of p-CREB in the hippocampus was also decreased $(P<0.05)$, while there was no significant difference between control groups and animals treated with Ro 20-1724 following ketamine exposure $(P>0.05)$. Electron microscopy demonstrated degenerative changes in hippocampal neurons of animals repetitively exposed to $70 \mathrm{mg} \cdot \mathrm{kg}^{-1}$ Ketamine, which was ameliorated by Ro 20-1724 $\left(0.5 \mathrm{mg} \cdot \mathrm{kg}^{-1}\right)$. Conclusion: The PDE-4 inhibitor Ro 20-1724 (0.5 $\left.\mathrm{mg} \cdot \mathrm{kg}^{-1}\right)$ reverses cognitive deficits associated with repeated ketamine exposure in young rats, most likely via increased cAMP/CREB signaling in the hippocampus and preservation of hippocampal neuronal ultrastructure.
\end{abstract}

Keywords: Research; Animal; Anesthesia; Neurodegeneration; Phsophodisterase Inhibitors; Memory

\section{Introduction}

Ketamine is an N-methyl-D-aspartate receptor (NMDA) antagonist, widely used in anesthesia. Pediatric burn patients who require multiple escharotomy procedures and dressing changes frequently undergo numerous and repeated ketmaine anesthetics as part of their medical care. Exposure of the developing brain to NMDA receptor antagonists, including ketamine, is associated with widespread neurodegeneration [1]. Previous studies show that ketamine, either following single or multiple exposures, may lead to cognitive impairment, including learning and memory ability in humans [1,2] and rodents [3-5]. Whe-

${ }^{*}$ Corresponding author. ther anesthetic exposure in childhood, particularly in humans, results in long-term learning disability and cognitive dysfunction is a matter of widespread debate. Most animal studies focus on recent cognitive function, with there being fewer studies of the long-term effects of anesthetic exposure in the literature. Therefore, this rat model was designed to examine long-term learning and memory behavior after repeated ketamine anesthesia in young rats.

Ro 20-1724 is a specific phosphodiesterase-4 (PDE-4) inhibitor. Recent studies show PDE-4 inhibitors can ameliorate cognitive dysfunction caused by sleep deprivation [6], $\mathrm{A} \beta$ protein exposure, neuroinflammation [7], intracerebroventricular streptozotocin injection [8], and 
neural ischemia [9]. This study examines whether Ro 20-1724 decreases long-term cognitive dysfunction following multiple ketamine exposures.

\section{Materials and Methods}

\subsection{Animals}

This study was approved by the Animal Use and Care Committee (AUCC) of Suzhou University. Sixty Sprague-Dawley rats, aged 21 days and weighing $60.0 \pm 5.0$ grams, were provided by the Laboratory Animal Center of Xuzhou Medical College. The housing and treatment of the animals was in accordance with institutional guidelines and approved by the AUCC. The mice were randomly divided into five groups of 12 individuals: a normal control group (C group), a $0.9 \%$ saline exposure group (S group), a ketamine exposure group (K group), a ketamine + Ro 20-1724 group (K + Ro group), and ketamine + vehicle group ( $\mathrm{K}+\mathrm{E}$ group) (the Ro 20-1724 is supplied in an ethanol vehicle). There were six individuals per group. Group K was anesthetized with $70 \mathrm{mg} / \mathrm{kg}$ intraperitoneal (ip) ketamine. Group $\mathrm{K}+\mathrm{R}$ received $0.5 \mathrm{mg} / \mathrm{kg}$ Ro 20-1724 (Sigma, USA) ip 30 minutes following the ketamine. Group $\mathrm{K}+\mathrm{E}$ received an equal volume of the ethanol vehicle instead of Ro 20-1724. Group S received saline only and group $\mathrm{C}$ received no treatment. The injections were administrated once daily for seven consecutive days. Following the series of injections, all animals were housed and fed under stamdard conditions until 8 weeks of age. On the first day of the ninth week, the Morris water maze was used to test cognitive ability, including a four-day consecutive place navigation test to evaluate learning function and a one-day spatial probe test to evaluate memory function. After completion of behavioral testing, the animals were sacrificed, their brains dissected and hippocampi rapidly removed for measurement of expression of phosphorylated cyclic AMP response element binding protein (p-CREB) by Western blot and examination of neuronal ultrastructure by transmission electron microscopy (TEM).

\subsection{Morris Water Maze Testing}

The Morris water maze (MWM) is a widely used tool to assess hippocampal-dependent learning and memory [10]. Evaluation of learning and memory was performed using the Morris water maze in a quiet, low-light environment at the same time every afternoon. The MWM consists on a black circular pool $(120 \mathrm{~cm}$ diameter, $60 \mathrm{~cm}$ high) filled with water $\left(30 \mathrm{~cm}\right.$ depth) at $25^{\circ} \mathrm{C} \pm 1{ }^{\circ} \mathrm{C}$ and virtually divided into four equivalent quadrants. An escape platform $(15 \mathrm{~cm}$ diameter, $30 \mathrm{~cm}$ high) was submerged $1.5 \mathrm{~cm}$ below the water surface and placed in the middle of one of the quadrants equidistant from the sidewall and the center of the pool.
1) Place navigation test: The Place navigation test was performed as previously described elsewhere [11]. Briefly, the animal was put into the MWM and forced to swim. When the animal finds the underwater platform, it gets a short break; if it is unable to climb onto the platform within 120 seconds, the animal is manually placed on the site and allowed to stay for $30 \mathrm{sec}-$ onds. In this study, the place navigation test was repeated over a period of four days. The decrease in latency from being placed in the MWM to finding the platform is a measurement of the animal's learning ability.

2) Spatial probe test: The spatial probe test was preformed as previously described elsewhere [12]. Briefly, once the animal has been trained to find the platform in the MWM, the platform is removed. The animal then tried to located the platform persistently based on memory acquired during training. The frequency of passing the platform's previous locating (FPP) is reflective of the animal's memory ability.

\subsection{Content of p-CREB Protein in Hippocampus by Western Blot}

After the behavior test, all animals were anesthetized by ip injection of chloral hydrate $(0.4 \mathrm{ml} / 100 \mathrm{~g})$, the thorax was opened and heart exposed. A tube was them placed through the left ventricle into the aorta and $200 \mathrm{ml}$ of saline was rapidly injected. $400 \mathrm{ml}$ of freshly prepared $4 \%$ paraformaldehyde in PBS buffer solution was then perfused at $4^{\circ} \mathrm{C}$.

The animals were then decapitated, the entire brain was removed and hippocampus quickly separated. In six animals from each group, a lysis buffer was added $(4 \mu \mathrm{l}: 1$ $\mathrm{mg}$ ) and the specimen was homogenized and centerfuged, with supernatant collected. p-CREB protein content was detected by bicinchonininc acid (BCA) assay. A 10\% sodium dodecyl sulfate-polyacrylamide gel electrophoresis (SDS-PAGE) was prepared according to the relative molecular mass of the target protein. The measured sample size was $70 \mu \mathrm{g}$. After concentration and electrophoretic separation process, the target protein was transmembrane separated for 53 minutes.

Western-blot (WB) hybridization: A $10 \mathrm{ml}$ washing buffer solution containing 3\% fetal bovine serum (BSA) was used to prepare the sample, gently shaking for 4 hours at room temperature. The first antibody of the CREB polyclonal (1:1000, Cell Signaling, USA) was added and the hybridization reaction proceeded at $4{ }^{\circ} \mathrm{C}$ overnight. The membrane was then washed three times, five minutes per wash, and rat anti-rabbit IgG secondary antibodies (1:1000, Beyotime Company, China) were added, gently shaking for 4 hours at room temperature. The membrane was again washed three times for 5 minutes per wash and 
the reaction was observed 15 minutes after adding colorimetric solution. The resulting color bands were then scanned and images saved. The expression of CREB protein was determined by semi-quantitative analysis of the gray scale ratio, using Image $\mathrm{J}$ analysis software.

\subsection{Electron Microscopy of Hippocampal Neurons}

In one animal from each group, after the brain was removed, the hippocampus was double fixed with glutaraldehyde-osmium tetroxide, epoxy-embedded, and cut into ultrathin sections. The hippocampal neurons were then observed by transmission electron microscopy $(\times 10,000)$.

\subsection{Statistical Analysis}

SPSS16.0 Software for was used for statistical analysis. Data are expressed as mean \pm standard deviation. Single factor analysis of variance (One-way ANOVA) was used for intergroup comparison. The least significant difference (LSD) test was analyzed between any two groups. A $\mathrm{p}$ value $<0.05$ was considered to be statistically significant.
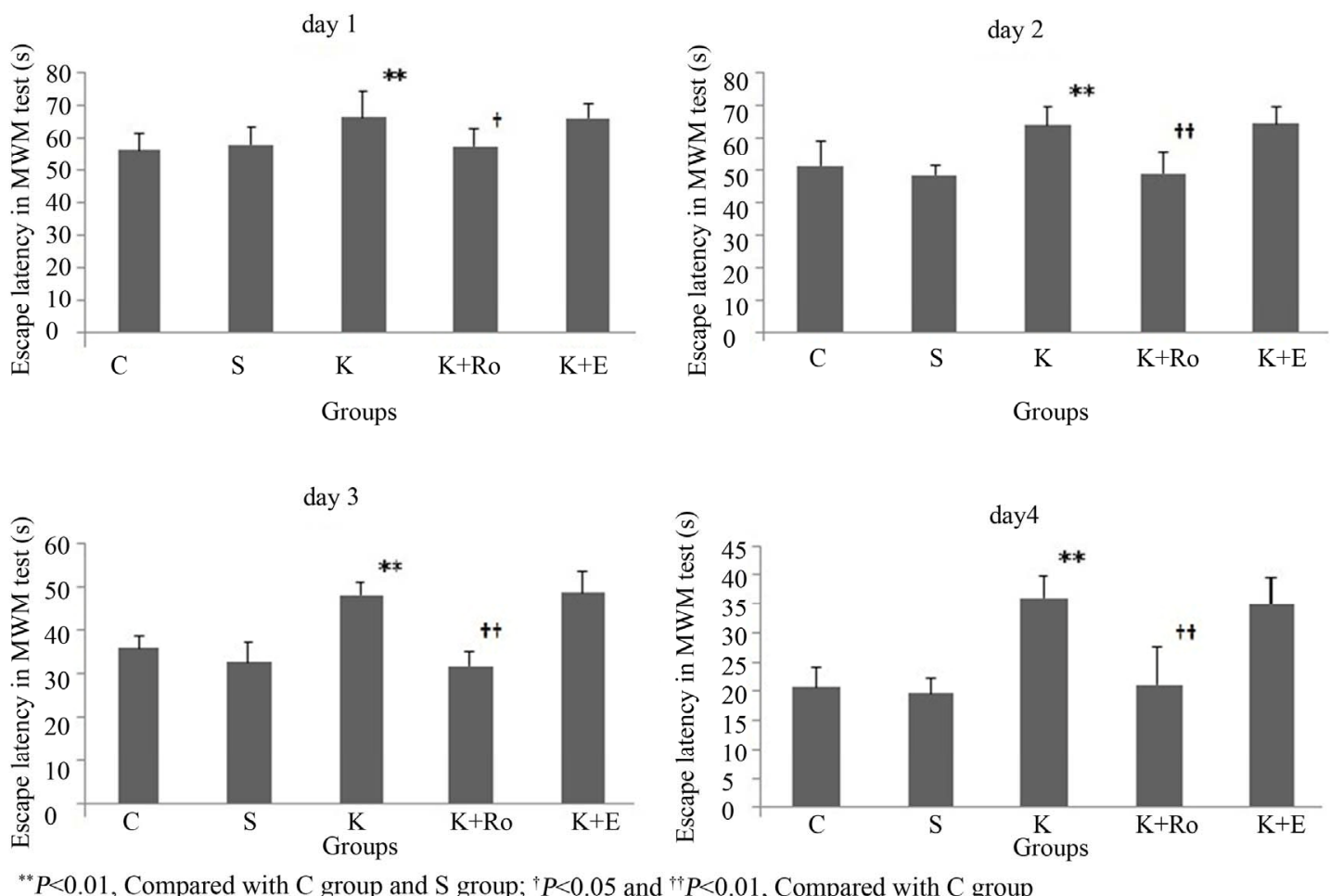

\section{Results}

\subsection{Place Navigation Test}

During the four-day process, escape latency gradually shortened in all animals and each animal could find the underwater platform in the end. The escape latency was significantly prolonged in the ketamine group $(\mathrm{K})$, compared with either normal controls (C) or animals that received only saline (S). In the animals treated with Ro 20-1724 $(K+R)$ the escape latency was significantly shorter than group $\mathrm{K}$ on the first day during the process $(P<0.05)$, and the difference continued to increase on days 2, 3 and $4(P<0.01)$. Both the saline and ketamine plus ethanol vehicle $(\mathrm{K}+\mathrm{E})$ groups demonstrated no effects, as there was no difference between either the $\mathrm{C}$ and $\mathrm{S}$ groups or the $\mathrm{K}$ and $\mathrm{K}+\mathrm{E}$ groups, respectively $(P>$ 0.05) (Figure 1).

\subsection{Spatial Probe Test}

The frequency of passing the platform (FPP) was significantly reduced in the $\mathrm{K}$ group, compared with $\mathrm{C}$ group $(P<0.05)$. Animals treated with Ro 20-1724 demonstrated significant improvement over the $\mathrm{K}$ group (Figures 2(a) and (b)).

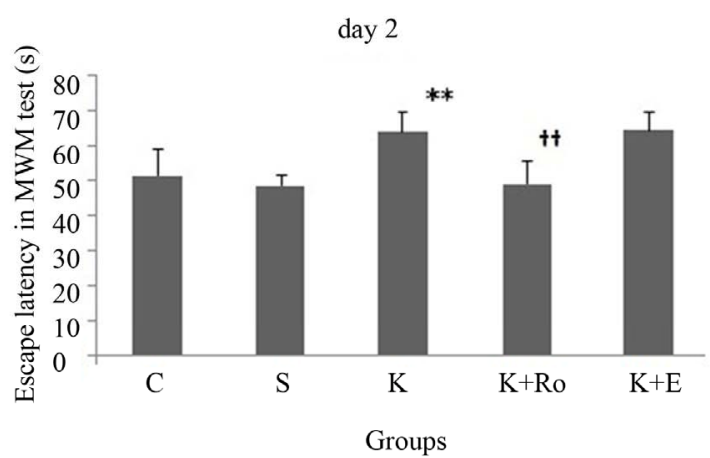

${ }^{* *} P<0.01$, Compared with $\mathrm{C}$ group and $\mathrm{S}$ group; ${ }^{\dagger} P<0.05$ and ${ }^{\dagger+} P<0.01$, Compared with $\mathrm{C}$ group

Figure 1. After seven consecutive days of anesthesia using ketamine on 21-day-old rats, the place navigation test was performed within the Morris water maze at 9 weeks of age. Over the learning course of four days, the animals that received ketamine anesthesia $(K)$, demonstrated longer escape latency in learning the procedure compared with normal controls $(P<$ 0.01) Animals treated with Ro 20-1724 $(K+R)$, showed significantly shorter escape latency compared to the $K$ group $(P<$ 0.01 or $P<0.05)$. Both saline and the ethanol vehicle had no effect. 


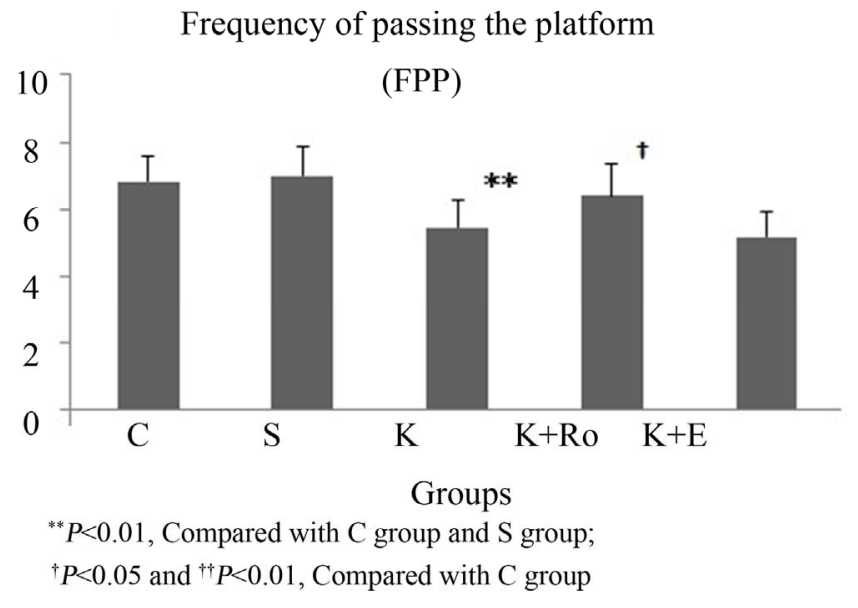

(a)

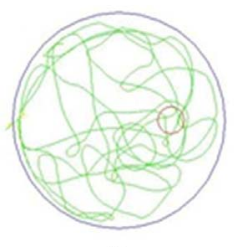

C

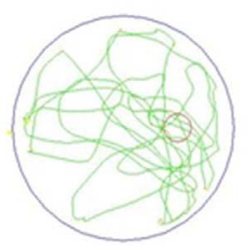

$\mathrm{K}+\mathrm{Ro}$

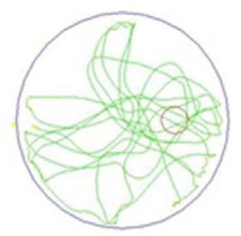

$\mathrm{S}$

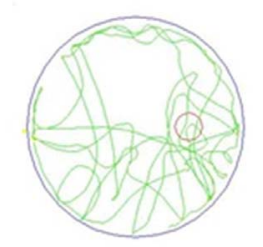

$\mathrm{K}+\mathrm{E}$

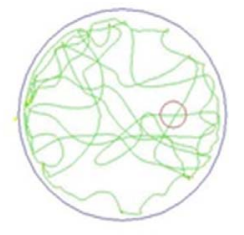

K

(b)

Figure 2. After four-days of training, all animals performed the spatial probe memory test. Frequency of passing the platform (FPP) was recorded to measure memory ability. The results shows significant reduction in the $\mathrm{K}$ group, compared with C group $(P<0.05)$; and after treatment by Ro $20-1724$, FPP was significant reversed. There are no differences between groups $C$ and $S$ or $K$ and $K+E$, respectively.

\subsection{Expression of p-CREB Protein in Hippocampus}

Expression of p-CREB protein determined by Western blot was significantly reduced in the $\mathrm{K}$ group $(P<0.05)$ and the decrease was reversed by Ro 20-1724 administration $(P<0.05)$. There were no significant differences between $\mathrm{C}$ group and $\mathrm{S}$ groups, or between the $\mathrm{K}$ and $\mathrm{K}$ + E groups $(P>0.05)$ (Figure 3$)$.

\subsection{Electron Microscopy}

The ultrastructure of hippocampal neurons in the CA1 area was observed by electron microscopy. In group $\mathrm{C}$ and group $\mathrm{S}$, large amounts of rough endoplasmic reticulum in hippocampal neuronal cytoplasm can be clearly observed. Ribosomes are well distributed, nuclear membrane integrity is preserved, and clear structure of neuritis can be seen. In group $\mathrm{K}$ and group $\mathrm{K}+\mathrm{E}$, hippocampal neurons demonstrated dramatic cell body swelling, the nuclear membrane appeared fractured, there was a lower density of ribosomes and degranulation of rough endoplasmic reticulum. In group $\mathrm{K}+\mathrm{Ro}$, hippocampal neurons showed only slight swelling, a slightly lower density of ribosomes and the nuclear membrane basic structure was maintained intact (Figure 4).

\section{Discussion}

Previous studies have shown that ketamine exposure in the immature brain can lead to cognitive dysfunction [15]. However, these studies are primarily concerned with short-term function and long-term studies in children are rare. Although much of the literature has focused on the developing brain, even in the adult, chronic ketamine

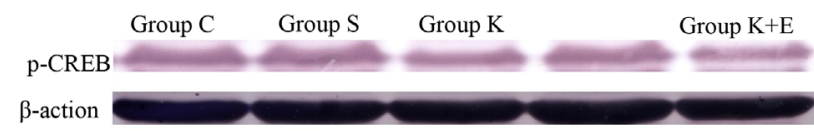

Figure 3. Expression of p-CREB protein was significantly reduced in the $K$ group $(P<0.05)$ and the decrease was reversed by $R o$ 20-1724 administration $(P<0.05)$.
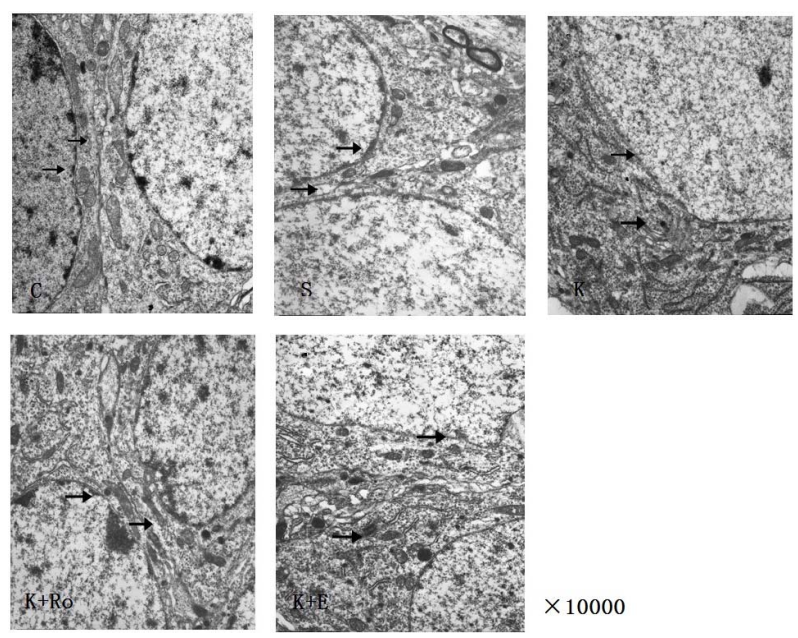

Figure 4. 10,000 $\times$ EM of neurons. Arrows indicate nuclear membrane structure, which was maintained by administration of Ro 20-1724.

exposure $(20 \mathrm{mg} / \mathrm{kg} /$ day $\times 14$ daily injections $)$ may lead to permanent cognitive change [13]. In neonatal rhesus monkeys, even one ketamine exposure for 24 hours, may cause long-lasting cognitive deficits [14]. The potential for ketamine anesthesia in childhood to affect cognitive function in adolescence has not been well studied.

This study confirmed that repeated ketamine anesthe- 
sia led to long-term learning and memory dysfunction, as measured by MWM testing. Escape latency represents the average time from the animal being placed into the pool to find the underwater platform. In the group exposed to ketamine, escape latency was significantly prolonged, suggesting that the learning deficits resulting from repeated ketamine anesthesia may last until adolescence. However, in animals that received Ro 20-1724 thirty minutes following each ketamine exposure, the increase in the escape latency was significantly rescued, especially on day 4 , when it was only about half that of the $\mathrm{K}$ group and close to normal, suggesting that Ro 20-1724 could ameliorate the learning deficits secondary to repeated ketamine exposure. These results parallel measurement of memory in the place probe test.

It appears, therefore, that repeated ketamine exposure in minor rats can result in learning and memory impairment that persists into adolescence, but the molecular events involved in this cognitive decline are poorly understood. Phosphodiesterase-4 (PDE4) is a specific intracellular high-affinity cAMP hydrolytic enzyme. PDE4 and its inhibitor can affect physiological function by regulating the intracellular cAMP levels. As a second messenger of signal transduction pathways in learning and memory [15-17], cAMP plays a pivotal role $[18,19]$. cAMP activates downstream protein kinase A (PKA) and protein kinase $\mathrm{C}$ (PKC) subunits entry to the nucleus, induces phosphorylation of specific serine stump site in cAMP response element binding protein (CREB) and activates CREB [20,21]. Phosphorylated CREB (pCREB) binds specifically to the cAMP response element (CRE) which, with cAMP regulates target genes in Deoxyribonucleic acid (DNA), starts downstream gene transcription and generates proteins believed necessary for learning and memory. PDE-4 can directly regulate the level of intracellular cAMP levels by hydrolysis, decrease phosphorylation of CREB, reduce activation and down-regulate relative gene expression that is controlled by CREB. This leads to proteins that participate in learning and memory not being synthesized, resulting in longterm learning and memory function impairment [22]. There are many members of the PDE-4 family. The most frequently studied drug is rolipram, which is developed as a potential treatment for depression and Parkinson's Disease. However, it was not introduced into clinical practice due to adverse effects of nausea and vomiting [23]. Therefore, we chose Ro 20-1724 as a research tool.

In our study, p-CREB expression in the hippocampus was analyzed by Western blot. In the ketamine group, p-CREB was significantly reduced after repeated exposure, however, when treated by Ro 20-1724, not only did behavior tasks of both learning and memory improvement, but also p-CREB expression in hippocampus was preserved. This suggests that Ro 20-1724 alleviates the impairment of learning and memory function caused by ketamine through enhancement of p-CREB expression in the hippocampus. Our study also demonstrated preservation of neuronal ultrastructure in the hippocampus on electron microscopy when animals exposed to ketamine were treated with Ro 20-1724. Yu et al. found that Ro 20-1724 can not only reverse ketamine-induced cognitive dysfunction, but can also shorten duration of anesthesia [24].

In summary, this study shows Ro 20-1724 can reverse the long-term learning and memory impairment caused by repeated ketamine exposure, possibly through enhanced p-CREB expression in the hippocampus. Specific PDE4 inhibitors, such as Ro 20-1724, may be promising agents for clinical applications, including the treatment of post-anesthesia cognitive dysfunction and further investigation is warranted.

\section{Acknowledgements}

This study was approved by the Animal Use and Care Committee (AUCC) of Suzhou University. This work was supported by The National Natural Science Foundation of China (Grant: 81000469) and Scientific Foundation from Health Office of Jiangsu province (H201070). The authors have no financial conflicts of interest.

\section{REFERENCES}

[1] J. H. Krystal, W. Abi-Saab, E. Perry, D. C. D’Souza, N. Liu, R. Gueorguieva, L. McDougall, T. Hunsberger, A. Belger, L. Levine and A. Breier, "Preliminary Evidence of Attenuation of the Disruptive Effects of the NMDA Glutamate Receptor Antagonist Ketamine on Working Memory by Pretreatment with the Group II Metabolic Glutamate Receptor Agonist LY354740 in Healthy Human Subjects," Psychopharmacology, Vol. 179, No. 1, 2005, pp. 303-309. doi:10.1007/s00213-004-1982-8

[2] C. J. Morgan, A. Mofeez, B. Brandner and H. V. Curran, "Acute Effects of Ketamine on Memory Systems and Psychotic Symptoms in Healthy Volunteers," Neuropsychopharmacology, Vol. 29, 2004, pp. 208-218. doi:10.1038/sj.npp.1300342

[3] J. H. Krystal, A. Bennett, D. Abi-Saab, A. Belger, L. P. Karper, D. C. D'Souza, D. Lipschitz, A. Abi-Dargham and D. S. Charney, "Dissociation of Ketamine Effects on Rule Acquisition and Rule Implementation: Possible Relevance to NMDA Receptor Contributions to Executive Cognitive Functions," Biological Psychiatry, Vol. 47, No. 2, 2000, pp. 137-143. doi:10.1016/S0006-3223(99)00097-9

[4] S. Peng, Y. Zhang, B. Ren, J. Zhang and J. H. Wang, "Effect of Ketamine Administration on Memory Consolidation, p-CREB and c-fos Expression in the Hippocampal Slices of Minor Rats," Molecular Biology Reports, Vol. 38, No. 4, 2011, pp. 2401-2407. 
doi:10.1007/s11033-010-0374-x

[5] S. Peng, Y. Zhang, H. Wang, B. Ren and J. Zhang, "Anesthetic Ketamine Counteracts Repetitive Mechanical StressInduced Learning and Memory Impairment in Developing Mice," Molecular Biology Reports, Vol. 38, No. 7, 2011, pp. 4347-4351. doi:10.1007/s11033-010-0561-9

[6] C. G. Vecsey, G. S. Baillie, D. Jaganath, R. Havekes, A. Daniels, M. Wimmer, T. Huang, K. M. Brown, X. Y. Li, G. Descalzi, S. S. Kim, T. Chen, Y. Z. Shang, M. Zhuo, M. D. Houslay and T. Abel, "Sleep Deprivation Impairs cAMP Signalling in the Hippocampus," Nature, Vol. 461, No. 7267, 2009, pp. 1122-1125. doi:10.1038/nature08488

[7] C. Wang, X. M. Yang, Y. Y. Zhuo, H. Zhou, H. B. Lin, Y. F. Cheng, J. P. Xu and H. T. Zhang, "The Phosphodiesterase-4 Inhibitor Rolipram Reverses A $\beta$-Induced Cognitive Impairment and Neuroinflammatory and Apoptotic Responses in Rats," International Journal of Neuropsychopharmacology, Vol. 15, No. 6, 2012, pp. 749-766. doi:10.1017/S1461145711000836

[8] V. Sharma, A. Bala, R. Deshmukh, K. L. Bedi and P. L. Sharma, "Neuroprotective Effect of RO-20-1724-a Phosphodiesterase4 Inhibitor against Intracerebroventricular Streptozotocin Induced Cognitive Deficit and Oxidative Stress in Rats," Pharmacology Biochemistry and Behavior, Vol. 101, No. 2, 2012, pp. 239-245. doi:10.1016/j.pbb.2012.01.004

[9] L. X. Li, Y. F. Cheng, H. B. Lin, C. Wang, J. P. Xu and H. T. Zhang, "Prevention of Cerebral Ischemia-Induced Memory Deficits by Inhibition of Phosphodiesterase-4 in Rats," Metabolic Brain Disease, Vol. 26, No. 1, 2011, pp. 37-47. doi:10.1007/s11011-011-9235-0

[10] V. K. Sharma, "Morris Water Maze: A Versatile Cognitive Tool," Journal of Bioscience and Technology, Vol. 1, 2009, pp. 15-19.

[11] R. Slamberová, M. Pometlová, L. Syllabová and M. Mancusková, "Learning in the Place Navigation Task, Not the New-Learning Task, Is Altered by Prenatal Methamphetamine Exposure," Developmental Brain Research, Vol. 157, No. 2, 2005, pp. 217-219.

doi:10.1016/j.devbrainres.2005.04.005

[12] R. G. M. Morris, “Morris Water Maze,” 2012. http://www.scholarpedia.org/article/Morris_water_maze

[13] R. E. Featherstone, Y. Liang, J. A. Saunders, V. M. TatardLeitman, R. S. Ehrlichman and S. J. Siegel, "Subchronic Ketamine Treatment Leads to Permanent Changes in EEG, Cognition and the Astrocytic Glutamate Transporter EAA T2 in Mice," Neurobiology of Disease, Vol. 47, No. 3, 2012, pp. 338-346. doi:10.1016/j.nbd.2012.05.003

[14] M. G. Paule, M. Li, R. R. Allen, F. Liu, X. Zou, C. Hotchkiss, J. P. Hanig, T. A. Patterson, W. Slikker Jr. and C. Wang, "Ketamine Anesthesia during the First Week of Life Can Cause Long-Lasting Cognitive Deficits in Rhesus Monkeys," Neurotoxicology and Teratology, Vol. 33, No. 2, 2011, pp. 220-230. doi:10.1016/j.ntt.2011.01.001
[15] E. R. Kandel and J. H. Schwartz, "Molecular Biology of Learning: Modulation of Transmitter Release," Science, Vol. 29, No. 4571, 1982, pp. 433-443. doi:10.1126/science.6289442

[16] E. R. Kandel, "The Molecular Biology of Memory Storage: A Dialogue between Genes and Synapses," Science, Vol. 294, No. 5544, 2001, pp. 1030-1038. doi:10.1126/science. 1067020

[17] X. Wang, L. Liu, S. Xia, C. Feng and A. Guo, "Relationship between Visual Learning/Memory Ability and Brain cAMP Level in Drosophila," Science in China Series C: Life Sciences, Vol. 4, 1998, pp. 503-511. doi:10.1007/BF02882888

[18] Y. F. Li, Y. Huang, S. L. Amsdell, L. Xiao, J. M. O’Donnell and H. T. Zhang, "Antidepressant- and AnxiolyticLike Effects of the Phosphodiesterase-4 Inhibitor Rolipram on Behavior Depend on Cyclic AMP Response Element Binding Protein-Mediated Neurogenesis in the Hippocampus," Neuropsychopharmacology, Vol. 34, 2009, pp. 2404-2419. doi:10.1038/npp.2009.66

[19] D. Jancic, M. Lopez de Armentia, L. M. Valor, R. Olivares and A. Barco, "Inhibition of cAMP Response Element-Binding Protein Reduces Neuronal Excitability and Plasticity, and Triggers Neurodegeneration," Cerebral Cortex, Vol. 19, No. 11, 2009, pp. 2535-2547. doi:10.1093/cercor/bhp004

[20] B. S. Skålhegg and K. Tasken, "Specificity in the cAMP/ PKA Signaling Pathway. Differential Expression Regulation, and Subcellular Localization of Subunit of PKA," Frontiers in Bioscience, Vol. 5, 2000, pp. 678-693. doi:10.2741/Skalhegg

[21] O. V. Vitolo, A. Sant'Angelo, V. Costanzo, F. Battaglia, O. Arancio and M. Shelanski, "Amyloid $\beta$-Peptide Inhibition of the PKA/CREB Pathway and Long-Term Potentiation: Reversibility by Drugs That Enhance cAMP Signaling," Proceedings of the National Academy of Sciences of USA, Vol. 99, No. 20, 2002, pp. 13217-13221. doi:10.1073/pnas.172504199

[22] A. Ghavami, W. D. Hirst and T. J. Novak, "Selective Phosphodiesterase (PDE)-4 Inhibitors: A Novel Approach to Treating Memory Deficit?" Drugs in R\&D, Vol. 7, 2006, pp. 63-71. doi:10.2165/00126839-200607020-00001

[23] V. Boswell-Smith, M. Cazzola and C. P. Page, "Are Phosphodiesterase 4 Inhibitors Just More Theophylline?" The Journal of Allergy and Clinical Immunology, Vol. 117, No. 6, 2006, pp. 1237-1243. doi:10.1016/j.jaci.2006.02.045

[24] M. C. Yu, J. H. Chen, C. Y. Lai, C. Y. Han and W. C. Ko, "Luteolin, a Non-Selective Competitive Inhibitor of Phosphodiesterases 1-5, Displaced [3H]-Rolipram from HighAffinity Rolipram Binding Sites and Reversed Xylazine/ Ketamine-Induced Anesthesia," European Journal of Pharmacology, Vol. 627, No. 1-3, 2010, pp. 269-275. doi:10.1016/j.ejphar.2009.10.031 